\title{
Incompressible Limit for a Two-Species Tumour Model with Coupling Through Brinkman's Law in One Dimension
}

\author{
Tomasz Dębiec ${ }^{1} \cdot$ Markus Schmidtchen $^{2}$
}

Received: 21 October 2019 / Accepted: 24 January 2020 / Published online: 4 February 2020

(C) The Author(s) 2020

\begin{abstract}
We present a two-species model with applications in tumour modelling. The main novelty is the coupling of both species through the so-called Brinkman law which is typically used in the context of visco-elastic media, where the velocity field is linked to the total population pressure via an elliptic equation. The same model for only one species has been studied by Perthame and Vauchelet in the past. The first part of this paper is dedicated to establishing existence of solutions to the problem, while the second part deals with the incompressible limit as the stiffness of the pressure law tends to infinity. Here we present a novel approach in one spatial dimension that differs from the kinetic reformulation used in the aforementioned study and, instead, relies on uniform BV-estimates.
\end{abstract}

Keywords Systems of PDEs · Mathematical biology · Tumour growth

\section{Introduction}

In recent years there has been an increasing interest in multi-phase models applied to tumour growth. Traditionally, tumour growth was modelled using a single equation describing the evolution of the abnormal cell density. This paper is dedicated to studying the two-species model

$$
\left\{\begin{aligned}
\frac{\partial n_{k}^{(i)}}{\partial t}-\nabla \cdot\left(n_{k}^{(i)} \nabla W_{k}\right) & =n_{k}^{(i)} G^{(i)}\left(p_{k}\right), \\
-v \Delta W_{k}+W_{k} & =p_{k},
\end{aligned}\right.
$$

T. Dębiec

t.debiec@mimuw.edu.pl

M. Schmidtchen

schmidtchen@1j11.math.upmc.fr

1 Institute of Applied Mathematics and Mechanics, University of Warsaw, Banacha 2, 02-097

Warsaw, Poland

2 Sorbonne Université, Laboratoire Jacques-Louis Lions, Paris 75005, France 
where $n^{(i)}$ represents the normal (resp. abnormal) cells, for $i=1,2$, and $k \in \mathbb{N}$ is a given constant modelling the stiffness of the total population pressure, $p_{k}$, which is generated by both species, i.e.,

$$
p_{k}:=\frac{k}{k-1}\left(n_{k}^{(1)}+n_{k}^{(2)}\right)^{k-1} .
$$

In addition, $v>0$ is a fixed positive constant that is understood as a measure of viscosity. The elliptic equation linking the macroscopic velocity potential, $W_{k}$, with the pressure $p_{k}$ is typically referred to as Brinkman's law, for instance $c f$. [1]. The growth of the two densities is assumed to be modulated by two functions $G^{(i)}$, for $i=1,2$, that are assumed to be decreasing in their variable, $p_{k}$, similar to $[7,20]$.

Throughout, we shall use the shorthand notation $n_{k}:=n_{k}^{(1)}+n_{k}^{(2)}$, in order to denote the total population. Upon adding up the two equations for the individual species, we obtain an equation for the total population density, $n_{k}$, i.e.,

$$
\frac{\partial n_{k}}{\partial t}-\nabla \cdot\left(n_{k} \nabla W_{k}\right)=n_{k}\left(r_{k} G^{(1)}\left(p_{k}\right)+\left(1-r_{k}\right) G^{(2)}\left(p_{k}\right)\right),
$$

where $r_{k}$ is the population fraction $r_{k}:=n_{k}^{(1)} / n_{k}$. Related models have been extensively studied in the past. We refer to [17, 19], and references therein, for a treatise of the incompressible limit for a single-species visco-elastic tumour model. As above, the velocity potential is given by an elliptic equation involving the pressure that, in their case, is just given by a power of the sole species. Introducing the coupling of the two equations for the individual species drastically changes the behaviour and the same tool employed in [19] cannot be applied, at least not in a straightforward manner, and a different strategy has to be found. Even in the case $v=0$ corresponding to the inviscid case, the system nature of the problem gives rise to a whole range of difficulties, $c f$. [6, 8, 13]. At first glance, the pressure gains in regularity, however, it gains just enough regularity to obtain compactness of its gradient, requiring a minute derivation of suitable estimates. Let us stress that the same type of difficulties are also encountered when the pressure is not given as a power law, $c f$. $[11,12,14]$. A key tool in obtaining existence results and stable (with respect to the parameter $k$ ) estimates is to devise and manipulate the equation satisfied by the (joint) population pressure, $c f$. [6, 8, 11-14, 16, 18, 19]. In this work we shall follow this path. An easy application of the chain rule in conjunction with Eq. (1) leads to

$$
\frac{\partial p_{k}}{\partial t}-\nabla p_{k} \cdot \nabla W_{k}=\frac{k-1}{v} p_{k}\left[W_{k}-p_{k}+v r_{k} G^{(1)}\left(p_{k}\right)+v\left(1-r_{k}\right) G^{(2)}\left(p_{k}\right)\right],
$$

where the population fraction $r_{k}$ satisfies

$$
\frac{\partial r_{k}}{\partial t}-\nabla r_{k} \cdot \nabla W_{k}=r_{k}\left(1-r_{k}\right)\left[G^{(1)}\left(p_{k}\right)-G^{(2)}\left(p_{k}\right)\right]
$$

The change to these new variables was first introduced in [2-4] in the context of a twospecies system where the two species avoid overcrowding. In a way, their works paved the way for more modern approaches to tumour models linked through Darcy's law, $c f$. $[5,6,8,13,18]$.

The rest of this paper is organised as follows. In the subsequent section we set up precisely the problem and state our assumptions. In Sect. 3 we establish existence of solutions to the main system under consideration, Eqs. (2a), (2b), and discuss their regularity necessary for our purposes. Section 4 is dedicated to establishing a range of a priori estimates 
necessary in the analysis of the incompressible limit. Section 5 is devoted to establishing the strong compactness of the pressure, which is key in passing to the stiff limit. Finally, with all information at hand, we pass to the incompressible limit in the pressure equation and derive the so-called complementarity relation in Sect. 6 . We round off the analytical results in Sect. 7 by presenting some numerical simulations for different parameter choices.

\section{Preliminaries and Statement of the Main Results}

We study the system

$$
\left\{\begin{array}{l}
\frac{\partial n_{k}^{(1)}}{\partial t}-\frac{\partial}{\partial x}\left(n_{k}^{(1)} \frac{\partial W_{k}}{\partial x}\right)=n_{k}^{(1)} G^{(1)}\left(p_{k}\right), \\
\frac{\partial n_{k}^{(2)}}{\partial t}-\frac{\partial}{\partial x}\left(n_{k}^{(2)} \frac{\partial W_{k}}{\partial x}\right)=n_{k}^{(2)} G^{(2)}\left(p_{k}\right),
\end{array}\right.
$$

posed on the whole domain $\mathbb{R}$. It is coupled through the Brinkman law

$$
-v \frac{\partial^{2}}{\partial x^{2}} W_{k}+W_{k}=p_{k}
$$

The system is equipped with non-negative initial data

$$
n_{0, k}^{(i)} \in L^{1}(\mathbb{R}) \cap L^{\infty}(\mathbb{R}),
$$

for any integer $k \geq 2$. Moreover, we assume that there exists a constant, $C>0$, such that

$$
\int_{\mathbb{R}}\left|\frac{\partial n_{0, k}^{(i)}}{\partial x}\right| \mathrm{d} x \leq C,
$$

for $i=1,2$, and every $k \geq 2$. As before, the pressure is given in form of a power of the joint population, i.e.,

$$
p_{k}:=\frac{k}{k-1}\left(n_{k}^{(1)}+n_{k}^{(2)}\right)^{k-1}=\frac{k}{k-1} n_{k}^{k-1} .
$$

Recall that the pressure satisfies

$$
\frac{\partial p_{k}}{\partial t}-\frac{\partial p_{k}}{\partial x} \frac{\partial W_{k}}{\partial x}=\frac{k-1}{v} p_{k}\left[W_{k}-p_{k}+v r_{k} G^{(1)}\left(p_{k}\right)+v\left(1-r_{k}\right) G^{(2)}\left(p_{k}\right)\right],
$$

with the population fraction, $r_{k}:=n_{k}^{(1)} / n_{k}$, given by

$$
\frac{\partial r_{k}}{\partial t}-\frac{\partial r_{k}}{\partial x} \frac{\partial W_{k}}{\partial x}=r_{k}\left(1-r_{k}\right)\left[G^{(1)}\left(p_{k}\right)-G^{(2)}\left(p_{k}\right)\right] .
$$

Throughout the paper we assume the following regularity and properties of the growth functions $G^{(i)}, i=1,2$,

$$
G^{(i)} \in C^{1}(\mathbb{R}), \quad G_{p}^{(i)} \leq-\alpha<0, \quad \text { as well as } G^{(i)}\left(p_{M}\right)=0,
$$


for some $p_{M}>0$, where $G_{p}^{(i)}$ denotes the derivative of the function $G^{(i)}$. The pressure $p_{M}$ is often called the homeostatic pressure.

Recall that a solution $W_{k}$ to Brinkman's equation $-v \partial_{x}^{2} W_{k}+W_{k}=p_{k}$ can be written as $W_{k}=K \star p_{k}$, where $K$ is the fundamental solution to the equation $-v \partial_{x}^{2} K+K=\delta_{0}$, i.e.,

$$
K(x)=\frac{1}{4 \pi} \int_{0}^{\infty} \exp \left[-\left(\pi|x|^{2} / 4 s v+s / 4 \pi\right)\right] s^{-1 / 2} \mathrm{~d} s=\frac{1}{2 \sqrt{v}} \exp \left(-v^{-1 / 2}|x|\right) .
$$

Then $K \geq 0, \int K(x) \mathrm{d} x=1$ and $K, \partial_{x} K \in L^{q}(\mathbb{R})$ for $1 \leq q \leq \infty$. By the elliptic regularity theory we have $W_{k}(t, \cdot) \in W^{2, q}(\mathbb{R})$, for any $t \in[0, T], 1 \leq q \leq \infty$.

Below we formulate the main results of this work.

Theorem 2.1 (Existence of Solutions) For any initial data satisfying (3a), (3b), system (2a), (2b) admits a unique solution $n_{k}^{(1)}, n_{k}^{(2)} \in L^{\infty}\left(0, T ; B V(\mathbb{R}) \cap L^{\infty}(\mathbb{R})\right)$.

We highlight the fact that solutions are essentially bounded since these bounds are not a consequence of the BV-bounds. Rather, they are obtained independently. This may prove useful for an extension to higher dimensions in future works.

Theorem 2.2 (Incompressible Limit and Complementarity Relation) We may pass to the limit $k \rightarrow \infty$ in the pressure equation, Eq. (4). This yields the so-called complementarity relation

$$
0=p_{\infty}\left(W_{\infty}-p_{\infty}+v n_{\infty}^{(1)} G^{(1)}\left(p_{\infty}\right)+v n_{\infty}^{(2)} G^{(2)}\left(p_{\infty}\right)\right)
$$

in the distributional sense, where $n_{\infty}^{(i)}, i=1,2$, satisfies

$$
\left\{\begin{aligned}
\frac{\partial n_{\infty}^{(i)}}{\partial t}-\frac{\partial}{\partial x}\left(n_{\infty}^{(i)} \frac{\partial W_{\infty}}{\partial x}\right) & =n_{\infty}^{(i)} G^{(i)}\left(p_{\infty}\right), \\
-v \frac{\partial^{2} W_{\infty}}{\partial x^{2}}+W_{\infty} & =p_{\infty} .
\end{aligned}\right.
$$

Moreover, the following holds true

$$
p_{\infty}\left(n_{\infty}-1\right)=0
$$

The subsequent sections are concerned with the proof of the two main theorems.

\section{Existence of Solutions and Regularity}

This section is dedicated to proving the existence of solutions to the $(p, r)$-system. The proof is based on an application of Banach's fixed point theorem. Let $k \geq 2$ be fixed throughout this section. Further, assume for now, that the initial data $u_{0}^{(i)}$ are Lipschitz continuous. For given functions $p, r \in L^{\infty}\left(0, T ; L^{\infty}(\mathbb{R})\right)$ we construct solutions $u^{(i)}$ to the linearised system, $i=1,2$,

$$
\frac{\partial u^{(i)}}{\partial t}-\frac{\partial u^{(i)}}{\partial x} \frac{\partial W}{\partial x}=\frac{k-1}{v} f^{(i)}(p, r),
$$


where

$$
f^{(1)}(p, r)=K \star p-p+v r G^{(1)}(p)+v(1-r) G^{(2)}(p)
$$

and

$$
f^{(2)}(p, r)=r(1-r)\left[G^{(1)}(p)-G^{(2)}(p)\right] .
$$

For the fixed $p$ from above, we may construct the backward flow

$$
\left\{\begin{aligned}
\frac{\mathrm{d} X_{(x, t)}}{\mathrm{d} s}(s) & =-\frac{\partial W}{\partial x}\left(X_{(x, t)}, s\right), \\
X_{(x, t)}(t) & =x
\end{aligned}\right.
$$

We readily observe that

$$
u^{(i)}(t, x)=u_{0}^{(i)}\left(X_{(x, t)}(s=0)\right)+\int_{0}^{t} f^{(i)}(p(\tau, x), r(\tau, x)) \mathrm{d} \tau,
$$

$i=1,2$, solve the linearised system (8). Now, considering another element $(\tilde{p}, \tilde{r})$ in $L^{\infty}\left(0, T ; L^{\infty}(\mathbb{R})\right)$, we observe that

$$
\begin{aligned}
\left|u^{(i)}(t, x)-\tilde{u}^{(i)}(t, x)\right| & =\left|u_{0}^{(i)}\left(X_{(x, t)}(s=0)\right)-u_{0}^{(i)}\left(\tilde{X}_{(x, t)}(s=0)\right)\right| \\
& \leq \operatorname{Lip}\left(u_{0}^{(i)}\right)\left|X_{(x, t)}(s=0)-\tilde{X}_{(x, t)}(s=0)\right| \\
& \leq \operatorname{Lip}\left(u_{0}^{(i)}\right) \int_{t}^{0}\left|\frac{\partial W}{\partial x}\left(X_{(x, t)}(s), s\right)-\frac{\partial \tilde{W}}{\partial x}\left(\tilde{X}_{(x, t)}(s), s\right)\right| \mathrm{d} s \\
& \leq \operatorname{Lip}\left(u_{0}^{(i)}\right) \int_{t}^{0}\left|\frac{\partial K}{\partial x} \star(p-\tilde{p})\right| \mathrm{d} s \\
& \leq \operatorname{Lip}\left(u_{0}^{(i)}\right) \int_{t}^{0}\left\|\frac{\partial K}{\partial x}\right\|_{L^{1}}\|p-\tilde{p}\|_{L^{\infty}} \mathrm{d} s \\
& \leq \operatorname{Lip}\left(u_{0}^{(i)}\right) T\left\|\frac{\partial K}{\partial x}\right\|_{L^{1}}\|p-\tilde{p}\|_{L^{\infty}} .
\end{aligned}
$$

Thus, upon passing to the supremum, we obtain the following stability estimate for two solutions

$$
\left\|u^{(i)}-\tilde{u}^{(i)}\right\|_{L^{\infty}} \leq C T\|p-\tilde{p}\|_{L^{\infty}} .
$$

In particular, for $T_{1}>0$ small enough the estimate gives rise to a contraction in the Banach space $L^{\infty}\left(0, T_{1} ; L^{\infty}(\mathbb{R})\right)$, which is sufficient to infer the existence of a unique fixed point, by an application of Banach's fixed point theorem. Since the supremum norm of the solution does not blow up, a finite number of iterations of the above argument leads to existence of a unique solution for all times $T>0$.

For the subsequent analysis, let us call this fixed point $\left(u_{*}^{(1)}, u_{*}^{(2)}\right)$. It remains to prove the expected BV-regularity of solutions. This is an easy consequence of the "transport nature" 
of the system, i.e.,

$$
\frac{\partial}{\partial t} \frac{\partial u_{*}^{(i)}}{\partial x}=\frac{\partial u_{*}^{(i)}}{\partial x} \frac{\partial W}{\partial x}+\frac{k-1}{v}\left[f_{p}^{(i)}\left(u_{*}^{(1)}, u_{*}^{(2)}\right) \frac{\partial u_{*}^{(1)}}{\partial x}+f_{r}^{(i)}\left(u_{*}^{(1)}, u_{*}^{(2)}\right) \frac{\partial u_{*}^{(2)}}{\partial x}\right] .
$$

Multiplying by $\operatorname{sign}\left(\partial_{x} u_{*}^{(i)}\right)$ and adding the two equations, for $i=1,2$, we obtain, after integrating

$$
\frac{\mathrm{d}}{\mathrm{d} t} \int_{\mathbb{R}}\left|\frac{\partial u_{*}^{(1)}}{\partial x}\right|+\left|\frac{\partial u_{*}^{(2)}}{\partial x}\right| \mathrm{d} x \leq C \int_{\mathbb{R}}\left|\frac{\partial u_{*}^{(1)}}{\partial x}\right|+\left|\frac{\partial u_{*}^{(2)}}{\partial x}\right| \mathrm{d} x,
$$

where the constant $C>0$ depends only on the Lipschitz constants of the functions $f^{(i)}$ and the $L^{\infty}$-bounds on the fixed point. In particular, from Gronwall's inequality we deduce a control on the BV-seminorm and, more importantly, the existence of solutions even in cases where $u_{0}^{(i)}$ is not Lipschitz continuous but only of bounded variation.

Using the fact that

$$
n^{(1)}=\left(\frac{k-1}{k} u_{*}^{(1)}\right)^{\frac{1}{k-1}} u_{*}^{(2)}, \quad \text { and } \quad n^{(2)}=\left(\frac{k-1}{k} u_{*}^{(1)}\right)^{\frac{1}{k-1}}\left(1-u_{*}^{(2)}\right),
$$

the existence and uniqueness result transfers to the original system for $n_{k}^{(i)}, i=1,2$.

Remark 3.1 (Extension to Higher Dimensions) Let us remark here that the same strategy can be easily extended to higher dimensions since the transport nature is the same in any dimension. In fact, the only "problematic" point in our strategy is the contraction argument which depends on $\left\|\partial_{x} K\right\|_{L^{1}}$. However, this norm is finite in any dimension, and therefore our existence result holds in any dimension.

\section{A Priori Estimates}

In this section we derive some bounds for the main quantities of interests, uniformly in $k$. These will be vital when passing to the limit with $k \rightarrow \infty$.

Lemma 4.1 (A priori estimates I) The following hold uniformly in $k$ for any $T>0$.

(i) $n_{k} \in L^{\infty}\left(0, T ; L^{1}(\mathbb{R})\right)$,

(ii) $p_{k} \in L^{\infty}\left(0, T ; L^{\infty}(\mathbb{R})\right)$,

(iii) $n_{k} \in L^{\infty}\left(0, T ; L^{\infty}(\mathbb{R})\right)$,

(iv) $p_{k} \in L^{\infty}\left(0, T ; L^{1}(\mathbb{R})\right)$, and

(v) $n_{k}^{(i)} \in L^{\infty}\left(0, T ; L^{\infty}(\mathbb{R})\right)$, for $i=1,2$.

Proof Clearly when $n_{k}(t=0) \geq 0$, then $n_{k}$ stays non-negative at all times. Integrating Eq. (1) in space and time we deduce that $n_{k} \in L^{\infty}\left(0, T ; L^{1}(\mathbb{R})\right)$ uniformly in $k$. By the maximum principle we have the bound $0 \leq p_{k} \leq p_{M}$. Then using $n_{k} \simeq p^{\frac{1}{k-1}}$ we deduce $n_{k} \in$ $L^{\infty}\left(0, T ; L^{\infty}(\mathbb{R})\right)$ uniformly. Writing $p_{k} \leq n_{k}\left\|n_{k}\right\|_{\infty}^{k-2}$ we see that $p_{k} \in L^{\infty}\left(0, T ; L^{1}(\mathbb{R})\right)$. Finally, we use that $n_{k}^{(1)}=r_{k} n_{k}$ and $0 \leq r_{k} \leq 1$ to deduce the last bounds.

Using the above Lemma and the boundedness of $W_{k}$, we have the following result. 
Lemma 4.2 (Integrability and Segregation) If both species are segregated initially, i.e.,

$$
\int_{\mathbb{R}} r_{k}^{0}\left(1-r_{k}^{0}\right) \mathrm{d} x=0
$$

then there holds

$$
\int_{\mathbb{R}} r_{k}(t, x)\left(1-r_{k}(t, x)\right) \mathrm{d} x=0,
$$

for all times $0 \leq t \leq T$. In particular, $r_{k}^{0}\left(1-r_{k}^{0}\right) \in L^{1}(\mathbb{R})$ implies $r_{k}\left(1-r_{k}\right) \in$ $L^{\infty}\left(0, T ; L^{1}(\mathbb{R})\right)$.

Proof Here and henceforth we shall employ the notation

$$
\left\|G^{(i)}\right\|_{\infty}:=\sup _{0 \leq p \leq P_{M}}\left|G^{(i)}(p)\right| .
$$

The supremum is taken only up to $p_{M}$, because in principle the functions $G^{(i)}$ can decrease arbitrarily. The uniform bound obtained in the previous proof shows however, that only the range $0 \leq p_{k} \leq p_{M}$ is relevant.

Using the equation for the population fraction and boundedness of the growth functions $G^{(i)}$, we obtain

$$
\begin{aligned}
\frac{\mathrm{d}}{\mathrm{d} t} \int_{\mathbb{R}} r_{k}\left(1-r_{k}\right) \mathrm{d} x & =\int_{\mathbb{R}}\left(1-2 r_{k}\right)\left(\frac{\partial r_{k}}{\partial x} \frac{\partial W_{k}}{\partial x}+r_{k}\left(1-r_{k}\right)\left[G^{(1)}\left(p_{k}\right)-G^{(2)}\left(p_{k}\right)\right]\right) \mathrm{d} x \\
& \leq \max _{i=1,2}\left\|G^{(i)}\right\|_{\infty} \int_{\mathbb{R}} r_{k}\left(1-r_{k}\right) \mathrm{d} x+\int_{\mathbb{R}} \frac{\partial}{\partial x}\left(r_{k}\left(1-r_{k}\right)\right) \frac{\partial W_{k}}{\partial x} \mathrm{~d} x \\
& \leq \max _{i=1,2}\left\|G^{(i)}\right\|_{\infty} \int_{\mathbb{R}} r_{k}\left(1-r_{k}\right) \mathrm{d} x-\int_{\mathbb{R}} r_{k}\left(1-r_{k}\right) \frac{\partial^{2} W_{k}}{\partial x^{2}} \mathrm{~d} x .
\end{aligned}
$$

Using Brinkman's law (2b), we obtain

$$
\begin{aligned}
\frac{\mathrm{d}}{\mathrm{d} t} \int_{\mathbb{R}} r_{k}\left(1-r_{k}\right) \mathrm{d} x & \leq \max _{i=1,2}\left\|G^{(i)}\right\|_{\infty} \int_{\mathbb{R}} r_{k}\left(1-r_{k}\right) \mathrm{d} x+\int_{\mathbb{R}} r_{k}\left(1-r_{k}\right) \frac{p_{k}-W_{k}}{v} \mathrm{~d} x \\
& \leq C \int_{\mathbb{R}} r_{k}\left(1-r_{k}\right) \mathrm{d} x,
\end{aligned}
$$

having used the a priori bounds on the pressure, $p_{k}$.

The following lemma establishes an $L^{1}$-bound on the right-hand side of the pressure equation.

Lemma 4.3 (A priori estimates II) The following estimate holds for any $T>0$

$$
k \int_{0}^{T} \int_{\mathbb{R}} p_{k}\left|W_{k}-p_{k}+v r_{k} G^{(1)}\left(p_{k}\right)+v\left(1-r_{k}\right) G^{(2)}\left(p_{k}\right)\right| \mathrm{d} x \mathrm{~d} t \leq C(T),
$$

for a constant $C(T)>0$, independent of $k$. Furthermore, the following bounds hold uniformly in $k$ : 
(i) $\frac{\partial W_{k}}{\partial t} \in L^{1}\left(0, T ; L^{q}(\mathbb{R})\right)$, for $1 \leq q \leq \infty$,

(ii) $\frac{\partial}{\partial t} \frac{\partial W_{k}}{\partial x} \in L^{1}\left(0, T ; L^{q}(\mathbb{R})\right)$, for $1<q<\infty$.

Proof Let us introduce the following notation

$$
Q_{k}:=W_{k}-p_{k}+v r_{k} G^{(1)}\left(p_{k}\right)+v\left(1-r_{k}\right) G^{(2)}\left(p_{k}\right),
$$

and follow the strategy of [19]. Using

$$
\frac{\partial W_{k}}{\partial t}=K \star\left[\frac{\partial p_{k}}{\partial x} \frac{\partial W_{k}}{\partial x}+\frac{k-1}{v} p_{k} Q_{k}\right],
$$

we derive the equation

$$
\begin{aligned}
\frac{\partial Q_{k}}{\partial t} & -\frac{\partial Q_{k}}{\partial x} \frac{\partial W_{k}}{\partial x}+\frac{k-1}{v} p_{k} Q_{k}\left[1-v r_{k} G_{p}^{(1)}\left(p_{k}\right)-v\left(1-r_{k}\right) G_{p}^{(2)}\left(p_{k}\right)\right] \\
& =-\left|\frac{\partial W_{k}}{\partial x}\right|^{2}+K \star\left[\frac{\partial p_{k}}{\partial x} \frac{\partial W_{k}}{\partial x}+\frac{k-1}{v} p_{k} Q_{k}\right]+v\left(G^{(1)}\left(p_{k}\right)-G^{(2)}\left(p_{k}\right)\right)^{2} r_{k}\left(1-r_{k}\right),
\end{aligned}
$$

and consequently,

$$
\begin{aligned}
& \frac{\partial\left|Q_{k}\right|}{\partial t}-\frac{\partial\left|Q_{k}\right|}{\partial x} \frac{\partial W_{k}}{\partial x}+\frac{k-1}{v} p_{k}\left|Q_{k}\right|\left[1-v r_{k} G_{p}^{(1)}\left(p_{k}\right)-v\left(1-r_{k}\right) G_{p}^{(2)}\left(p_{k}\right)\right] \\
& \leq-\left|\frac{\partial W_{k}}{\partial x}\right|^{2}+\left|K \star\left[\frac{\partial p_{k}}{\partial x} \frac{\partial W_{k}}{\partial x}+\frac{k-1}{v} p_{k}\left|Q_{k}\right|\right]\right| \\
& \quad+v\left(G^{(1)}\left(p_{k}\right)-G^{(2)}\left(p_{k}\right)\right)^{2} r_{k}\left(1-r_{k}\right) .
\end{aligned}
$$

Integrating in space-time and using the assumption that $\left|G_{p}^{(i)}\right| \geq \alpha>0$, we obtain

$$
\begin{aligned}
\alpha(k-1) \int_{0}^{T} \int_{\mathbb{R}} p_{k}\left|Q_{k}\right| \mathrm{d} x \mathrm{~d} t \leq & \int_{\mathbb{R}}\left|Q_{k}(x, 0)\right|-\left|Q_{k}(x, T)\right| \mathrm{d} x+\int_{0}^{T} \int_{\mathbb{R}}\left|\frac{\partial W_{k}}{\partial x}\right|^{2} \mathrm{~d} x \mathrm{~d} t \\
& +\int_{0}^{T} \int_{\mathbb{R}} v^{-1}\left|Q_{k}\right|\left(p_{k}-W_{k}\right)+\left|K \star\left[\frac{\partial p_{k}}{\partial x} \frac{\partial W_{k}}{\partial x}\right]\right| \mathrm{d} x \mathrm{~d} t \\
& +\int_{0}^{T} \int_{\mathbb{R}} v\left(G^{(1)}\left(p_{k}\right)-G^{(2)}\left(p_{k}\right)\right)^{2} r_{k}\left(1-r_{k}\right) \mathrm{d} x \mathrm{~d} t .
\end{aligned}
$$

The first three terms on the right-hand side are controlled uniformly, as is the very last term. For the remaining two terms we write

$$
v^{-1} \int_{0}^{T} \int_{\mathbb{R}}\left|Q_{k}\right|\left(p_{k}-W_{k}\right) \mathrm{d} x \mathrm{~d} t \leq v^{-1} \int_{0}^{T} \int_{\mathbb{R}}\left|Q_{k}\right| p_{k} \mathrm{~d} x \mathrm{~d} t,
$$

which, for $k$ large enough, is controlled by the left-hand side of the last inequality, and

$$
\begin{aligned}
K \star\left[\frac{\partial p_{k}}{\partial x} \frac{\partial W_{k}}{\partial x}\right] & =\frac{\partial K}{\partial x} \star\left[p_{k} \frac{\partial W_{k}}{\partial x}\right]-K \star\left[p_{k} \frac{\partial^{2} W_{k}}{\partial x^{2}}\right] \\
& =\frac{\partial K}{\partial x} \star\left[p_{k} \frac{\partial K}{\partial x} \star p_{k}\right]-K \star\left[p_{k} \frac{\partial^{2} W_{k}}{\partial x^{2}}\right] .
\end{aligned}
$$


Using Lemma 4.1, we see that the right-hand side is uniformly bounded in $L^{\infty}\left(0, T ; L^{q}(\mathbb{R})\right)$, $1 \leq q \leq \infty$. It follows that

$$
\alpha(k-1) \int_{0}^{T} \int_{\mathbb{R}} p_{k}\left|Q_{k}\right| \mathrm{d} x \mathrm{~d} t \leq C(T),
$$

as desired.

Now, using Eq. (9) and the above computations, it is clear that $\partial_{t} W_{k}$ is uniformly bounded in $L^{\infty}\left(0, T ; L^{q}(\mathbb{R})\right)$, for $1 \leq q \leq \infty$. Finally we write

$$
\frac{\partial}{\partial t} \frac{\partial W_{k}}{\partial x}=\frac{\partial^{2} K}{\partial x^{2}} \star\left(p_{k} \frac{\partial W_{k}}{\partial x}\right)-\frac{\partial K}{\partial x} \star\left(p_{k} \frac{\partial^{2} W_{k}}{\partial x^{2}}\right)+\frac{k-1}{v} \frac{\partial K}{\partial x} \star\left(p_{k} Q_{k}\right),
$$

and use the definition of $K, c f$. Eq. (7), to conclude the proof.

Remark 4.4 All the results of this section remain valid in any spatial dimension $d \geq 1$, see for example [19] for the a priori estimates, and the $L^{1}$-bound on the quantity $k p_{k} Q_{k}$.

\section{Strong Compactness of the Pressure}

This section is solely dedicated to the derivation of suitable estimates in order to obtain strong compactness of the pressure, $p_{k}$. A key step in this pursuit is the following BVestimate on the individual species as well as the total population.

Lemma 5.1 (Regularity of $n_{k}^{(i)}$ and $n_{k}$ ) For $i=1,2$, we have the following

$$
\left|\frac{\partial n_{k}^{(i)}}{\partial x}\right|,\left|\frac{\partial n_{k}}{\partial x}\right| \in L^{\infty}\left(0, T ; L^{1}(\mathbb{R})\right),
$$

uniformly in $k \geq 2$.

Proof For $i=1,2$, we consider

$$
\frac{\partial n_{k}^{(i)}}{\partial t}=\frac{\partial}{\partial x}\left(n_{k}^{(i)} \frac{\partial W_{k}}{\partial x}\right)+n_{k}^{(i)} G^{(i)}\left(p_{k}\right)
$$

Upon differentiating in space, we obtain

$$
\frac{\partial}{\partial t} \frac{\partial n_{k}^{(i)}}{\partial x}=\frac{\partial}{\partial x}\left(\frac{\partial n_{k}^{(i)}}{\partial x} \frac{\partial W}{\partial x}\right)+\frac{\partial}{\partial x}\left(n_{k}^{(i)} \frac{\partial^{2} W}{\partial x^{2}}\right)+\frac{\partial n_{k}^{(i)}}{\partial x} G^{(i)}\left(p_{k}\right)+n_{k}^{(i)} G_{p}^{(i)}\left(p_{k}\right) \frac{\partial p_{k}}{\partial x},
$$

for $i=1,2$. Upon adding up both equations we get

$$
\frac{\partial}{\partial t} \frac{\partial n_{k}}{\partial x}=\frac{\partial}{\partial x}\left(\frac{\partial n_{k}}{\partial x} \frac{\partial W_{k}}{\partial x}\right)+\frac{\partial}{\partial x}\left(n_{k} \frac{\partial^{2} W_{k}}{\partial x^{2}}\right)+\sum_{i=1,2} \frac{\partial n_{k}^{(i)}}{\partial x} G^{(i)}\left(p_{k}\right)+n_{k}^{(i)} G_{p}^{(i)}\left(p_{k}\right) \frac{\partial p_{k}}{\partial x} .
$$


Multiplying the equation for the individual species by $\sigma^{(i)}:=\operatorname{sign}\left(\partial_{x} n_{k}^{(i)}\right)$ and the equation for the total population by $\sigma:=\operatorname{sign}\left(\partial_{x} n_{k}\right)$, we get, upon adding the two equations in Eq. (10) and the one in Eq. (11), and integrating in space

$$
\begin{aligned}
\frac{\mathrm{d}}{\mathrm{d} t} \int_{\mathbb{R}}\left|\frac{\partial n_{k}^{(1)}}{\partial x}\right| & +\left|\frac{\partial n_{k}^{(2)}}{\partial x}\right|+\left|\frac{\partial n_{k}}{\partial x}\right| \mathrm{d} x \\
= & \int_{\mathbb{R}} \frac{\partial}{\partial x}\left(\left|\frac{\partial n_{k}^{(1)}}{\partial x}\right| \frac{\partial W_{k}}{\partial x}\right)+\frac{\partial}{\partial x}\left(n_{k}^{(i)} \frac{\partial^{2} W_{k}}{\partial x^{2}}\right) \sigma^{(1)}+\left|\frac{\partial n_{k}^{(1)}}{\partial x}\right| G^{(1)}\left(p_{k}\right) \\
& +\sigma^{(1)} n_{k}^{(1)} G_{p}^{(1)}\left(p_{k}\right) \frac{\partial p_{k}}{\partial x} \\
+ & \frac{\partial}{\partial x}\left(\left|\frac{\partial n_{k}^{(2)}}{\partial x}\right| \frac{\partial W_{k}}{\partial x}\right)+\frac{\partial}{\partial x}\left(n_{k}^{(2)} \frac{\partial^{2} W_{k}}{\partial x^{2}}\right) \sigma^{(2)}+\left|\frac{\partial n_{k}^{(2)}}{\partial x}\right| G^{(2)}\left(p_{k}\right) \\
& +\sigma^{(2)} n_{k}^{(2)} G_{p}^{(2)}\left(p_{k}\right) \frac{\partial p_{k}}{\partial x} \\
+ & \frac{\partial}{\partial x}\left(\left|\frac{\partial n_{k}}{\partial x}\right| \frac{\partial W_{k}}{\partial x}\right)+\frac{\partial}{\partial x}\left(n_{k} \frac{\partial^{2} W_{k}}{\partial x^{2}}\right) \sigma+\sum_{i=1,2} \sigma \frac{\partial n_{k}^{(i)}}{\partial x} G^{(i)}\left(p_{k}\right) \\
& +n_{k}^{(i)} G_{p}^{(i)}\left(p_{k}\right)\left|\frac{\partial p_{k}}{\partial x}\right| \mathrm{d} x .
\end{aligned}
$$

First we notice that the exact derivatives vanish and the estimate simplifies to

$$
\begin{aligned}
\frac{\mathrm{d}}{\mathrm{d} t} \int_{\mathbb{R}}\left|\frac{\partial n_{k}^{(1)}}{\partial x}\right|+\left|\frac{\partial n_{k}^{(2)}}{\partial x}\right|+\left|\frac{\partial n_{k}}{\partial x}\right| \mathrm{d} x \\
\leq \int_{\mathbb{R}} \frac{\partial}{\partial x}\left(n_{k}^{(1)} \frac{\partial^{2} W_{k}}{\partial x^{2}}\right) \sigma^{(1)}+\left|\frac{\partial n_{k}^{(1)}}{\partial x}\right| G^{(1)}\left(p_{k}\right)+n_{k}^{(1)}\left|G_{p}^{(1)}\left(p_{k}\right)\right|\left|\frac{\partial p_{k}}{\partial x}\right| \\
+\frac{\partial}{\partial x}\left(n_{k}^{(2)} \frac{\partial^{2} W_{k}}{\partial x^{2}}\right) \sigma^{(2)}+\left|\frac{\partial n_{k}^{(2)}}{\partial x}\right| G^{(2)}\left(p_{k}\right)+n_{k}^{(2)}\left|G_{p}^{(2)}\left(p_{k}\right)\right|\left|\frac{\partial p_{k}}{\partial x}\right| \\
+\frac{\partial}{\partial x}\left(n_{k} \frac{\partial^{2} W_{k}}{\partial x^{2}}\right) \sigma+\sum_{i=1,2}\left|\frac{\partial n_{k}^{(i)}}{\partial x}\right|\left\|G^{(i)}\right\|_{\infty}+n_{k}^{(i)} G_{p}^{(i)}\left(p_{k}\right)\left|\frac{\partial p_{k}}{\partial x}\right| \mathrm{d} x .
\end{aligned}
$$

Next, we notice that all the terms involving the pressure gradient cancel due to opposite signs, whence

$$
\begin{aligned}
\frac{\mathrm{d}}{\mathrm{d} t} \int_{\mathbb{R}}\left|\frac{\partial n_{k}^{(1)}}{\partial x}\right|+\left|\frac{\partial n_{k}^{(2)}}{\partial x}\right|+\left|\frac{\partial n_{k}}{\partial x}\right| \mathrm{d} x \\
\quad \leq \int_{\mathbb{R}} \frac{\partial}{\partial x}\left(n_{k}^{(1)} \frac{\partial^{2} W_{k}}{\partial x^{2}}\right) \sigma^{(1)}+\left|\frac{\partial n_{k}^{(1)}}{\partial x}\right| G^{(1)}\left(p_{k}\right)
\end{aligned}
$$




$$
\begin{aligned}
& +\frac{\partial}{\partial x}\left(n_{k}^{(2)} \frac{\partial^{2} W_{k}}{\partial x^{2}}\right) \sigma^{(2)}+\left|\frac{\partial n_{k}^{(2)}}{\partial x}\right| G^{(2)}\left(p_{k}\right) \\
& +\frac{\partial}{\partial x}\left(n_{k} \frac{\partial^{2} W_{k}}{\partial x^{2}}\right) \sigma+\sum_{i=1,2}\left|\frac{\partial n_{k}^{(i)}}{\partial x}\right|\left\|G^{(i)}\right\|_{\infty} \mathrm{d} x .
\end{aligned}
$$

Thus we are left with

$$
\begin{aligned}
\frac{\mathrm{d}}{\mathrm{d} t} \int_{\mathbb{R}}\left|\frac{\partial n_{k}^{(1)}}{\partial x}\right|+\left|\frac{\partial n_{k}^{(2)}}{\partial x}\right|+\left|\frac{\partial n_{k}}{\partial x}\right| \mathrm{d} x \\
\leq C \int_{\mathbb{R}}\left|\frac{\partial n_{k}^{(1)}}{\partial x}\right|+\left|\frac{\partial n_{k}^{(2)}}{\partial x}\right|+\left|\frac{\partial n_{k}}{\partial x}\right| \mathrm{d} x \\
\quad+\int_{\mathbb{R}} \frac{\partial}{\partial x}\left(n_{k}^{(1)} \frac{\partial^{2} W_{k}}{\partial x^{2}}\right) \sigma^{(1)}+\frac{\partial}{\partial x}\left(n_{k}^{(2)} \frac{\partial^{2} W_{k}}{\partial x^{2}}\right) \sigma^{(2)}+\frac{\partial}{\partial x}\left(n_{k} \frac{\partial^{2} W_{k}}{\partial x^{2}}\right) \sigma \mathrm{d} x .
\end{aligned}
$$

Using the fact that

$$
-v \frac{\partial^{2} W_{k}}{\partial x^{2}}+W_{k}=p_{k}
$$

the integrand of the last line of Eq. (12) may be simplified to

$$
\begin{aligned}
& \frac{\partial}{\partial x}\left(n_{k}^{(1)} \frac{\partial^{2} W_{k}}{\partial x^{2}}\right) \sigma^{(1)}+\frac{\partial}{\partial x}\left(n_{k}^{(2)} \frac{\partial^{2} W_{k}}{\partial x^{2}}\right) \sigma^{(2)}+\frac{\partial}{\partial x}\left(n_{k} \frac{\partial^{2} W_{k}}{\partial x^{2}}\right) \sigma \\
& =v^{-1}\left|\frac{\partial n_{k}^{(1)}}{\partial x}\right|\left(W_{k}-p_{k}\right)+v^{-1} n_{k}^{(1)} \frac{\partial}{\partial x}\left(W_{k}-p_{k}\right) \sigma^{(1)} \\
& +v^{-1}\left|\frac{\partial n_{k}^{(2)}}{\partial x}\right|\left(W_{k}-p_{k}\right)+v^{-1} n_{k}^{(2)} \frac{\partial}{\partial x}\left(W_{k}-p_{k}\right) \sigma^{(2)} \\
& +v^{-1}\left|\frac{\partial n_{k}}{\partial x}\right|\left(W_{k}-p_{k}\right)+v^{-1} n_{k} \frac{\partial}{\partial x}\left(W_{k}-p_{k}\right) \sigma .
\end{aligned}
$$

Using the fact that $\left|\sigma^{(i)}\right|,|\sigma| \leq 1$ and exploiting the bounds

$$
n_{k}^{(i)}, n_{k} \in L^{\infty}\left(0, T ; L^{1}(\mathbb{R})\right), \quad \text { and } \quad \frac{\partial W_{k}}{\partial x} \in L^{\infty}\left(0, T ; L^{\infty}(\mathbb{R})\right),
$$

we may bound the terms of Eq. (13), and the last line of Eq. (12) becomes

$$
\begin{gathered}
\int_{\mathbb{R}} \frac{\partial}{\partial x}\left(n_{k}^{(1)} \frac{\partial^{2} W_{k}}{\partial x^{2}}\right) \sigma^{(1)}+\frac{\partial}{\partial x}\left(n_{k}^{(2)} \frac{\partial^{2} W_{k}}{\partial x^{2}}\right) \sigma^{(2)}+\frac{\partial}{\partial x}\left(n_{k} \frac{\partial^{2} W_{k}}{\partial x^{2}}\right) \sigma \mathrm{d} x \\
=C v^{-1} \int_{R}\left|\frac{\partial n_{k}^{(1)}}{\partial x}\right|+\left|\frac{\partial n_{k}^{(2)}}{\partial x}\right|+\left|\frac{\partial n_{k}}{\partial x}\right| \mathrm{d} x \\
+C+v^{-1} \int_{\mathbb{R}} n_{k}^{(1)}\left|\frac{\partial p_{k}}{\partial x}\right|+n_{k}^{(2)}\left|\frac{\partial p_{k}}{\partial x}\right|-n_{k}\left|\frac{\partial p_{k}}{\partial x}\right| \mathrm{d} x .
\end{gathered}
$$


The last integral in Eq. (14) vanishes due to the fact that $n_{k}=n_{k}^{(1)}+n_{k}^{(2)}$. Thus, substituting Eq. (14) into Eq. (12), an application of Gronwall's lemma yields the BV-estimate in space.

Corollary 5.2 From the proof of the preceding lemma we deduce

$$
\int_{0}^{T} \int_{\mathbb{R}} n_{k}\left|\frac{\partial p_{k}}{\partial x}\right| \mathrm{d} x \mathrm{~d} t \leq C,
$$

where $C>0$ is independent of $k$.

Proof Let us revisit the equation for $\partial_{t} n_{k}$, i.e.,

$$
\begin{aligned}
\frac{\mathrm{d}}{\mathrm{d} t} \int_{\mathbb{R}}\left|\frac{\partial n_{k}}{\partial x}\right| \mathrm{d} x \leq & C+C \int_{\mathbb{R}}\left|\frac{\partial n_{k}^{(1)}}{\partial x}\right|+\left|\frac{\partial n_{k}^{(2)}}{\partial x}\right|+\left|\frac{\partial n_{k}}{\partial x}\right| \mathrm{d} x \\
& +\int_{\mathbb{R}}\left(n_{k}^{(1)} G_{p}^{(1)}\left(p_{k}\right)+n_{k}^{(2)} G_{p}^{(2)}\left(p_{k}\right)-v^{-1} n_{k}\right)\left|\frac{\partial p_{k}}{\partial x}\right| \mathrm{d} x .
\end{aligned}
$$

Now we use the bounds $G_{p}^{(i)} \leq-\alpha<0$, for $i=1,2$, and integrate in time to see that

$$
\left(v^{-1}+\alpha\right) \int_{0}^{T} \int_{\mathbb{R}} n_{k}\left|\frac{\partial p_{k}}{\partial x}\right| \mathrm{d} x \mathrm{~d} t \leq 2\left\|\frac{\partial n_{k}}{\partial x}\right\|_{L_{\left(0, T ; L^{1}(\mathbb{R})\right)}}+C T R,
$$

where

$$
R:=\left\|\frac{\partial n_{k}}{\partial x}\right\|_{L^{\infty}\left(0, T ; L^{1}(\mathbb{R})\right)}+\left\|\frac{\partial n_{k}^{(1)}}{\partial x}\right\|_{L^{\infty}\left(0, T ; L^{1}(\mathbb{R})\right)}+\left\|\frac{\partial n_{k}^{(2)}}{\partial x}\right\|_{L^{\infty}\left(0, T ; L^{1}(\mathbb{R})\right)}+1 .
$$

Thus we infer that $n_{k} \partial_{x} p_{k}$ is uniformly bounded in $L^{1}\left(0, T ; L^{1}(\mathbb{R})\right)$.

Lemma 5.3 (Strong Compactness of the Pressure) There exists a function

$$
p_{\infty} \in L^{\infty}\left(0, T ; L^{1}(\mathbb{R}) \cap L^{\infty}(\mathbb{R})\right),
$$

such that, up to a subsequence, there holds

$$
p_{k} \longrightarrow p_{\infty}
$$

in $L_{\mathrm{loc}}^{p}\left(0, T ; L^{q}(\mathbb{R})\right)$, as $k \rightarrow \infty$ for any $2 \leq p, q<\infty$. In addition, the convergence also holds in the almost everywhere sense.

Proof Let us write the quantity $n_{k}\left|\partial_{x} p_{k}\right|$ as a spatial derivative of a non-decreasing function of the pressure. We compute as follows

$$
n_{k}\left|\frac{\partial p_{k}}{\partial x}\right|=\left(\frac{k-1}{k}\right)^{\frac{k}{k-1}} \operatorname{sign}\left(\frac{\partial p_{k}}{\partial x}\right) \frac{\partial}{\partial x}\left(p^{\frac{k}{k-1}}\right)=\left(\frac{k-1}{k}\right)^{\frac{k}{k-1}}\left|\frac{\partial}{\partial x}\left(p^{\frac{k}{k-1}}\right)\right| .
$$

Let $\phi_{k}(z):=z^{\frac{k}{k-1}}$. Then

$$
\int_{0}^{T} \int_{\mathbb{R}} n_{k}\left|\frac{\partial p_{k}}{\partial x}\right| \mathrm{d} x \mathrm{~d} t \geq \frac{1}{4} \int_{0}^{T} \int_{\mathbb{R}}\left|\frac{\partial \phi_{k}}{\partial x}\left(p_{k}\right)\right| \mathrm{d} x \mathrm{~d} t
$$


i.e., $\partial_{x} \phi_{k}\left(p_{k}\right) \in L^{1}\left(0, T ; L^{1}(\mathbb{R})\right)$, uniformly in $k$. Moreover, we have the same $L^{1}$-bound for the time derivative of $\phi_{k}\left(p_{k}\right)$. Indeed

$$
\begin{aligned}
\frac{\partial \phi_{k}}{\partial t}\left(p_{k}\right) & =\phi_{k}^{\prime}\left(p_{k}\right) \frac{\partial p_{k}}{\partial t} \\
& =\phi_{k}^{\prime}\left(p_{k}\right)\left(\frac{\partial p_{k}}{\partial x} \frac{\partial W_{k}}{\partial x}+\frac{k-1}{v} p_{k} Q_{k}\right) \\
& =\frac{\partial \phi_{k}}{\partial x}\left(p_{k}\right) \frac{\partial W_{k}}{\partial x}+\frac{k}{k-1} \frac{k-1}{v} p_{k}^{\frac{1}{k-1}} p_{k} Q_{k},
\end{aligned}
$$

and therefore

$$
\begin{aligned}
\int_{0}^{T} \int_{\mathbb{R}}\left|\frac{\partial \phi_{k}}{\partial t}\left(p_{k}\right)\right| \mathrm{d} x \mathrm{~d} t & \leq\left\|\frac{\partial \phi_{k}}{\partial t}\left(p_{k}\right)\right\|_{L^{1}\left(0, T ; L^{1}(\mathbb{R})\right)}\left\|\frac{\partial W}{\partial x}\right\|_{L^{\infty}\left(0, T ; L^{\infty}(\mathbb{R})\right)} \\
& +2 p_{M}^{\frac{1}{k-1}} \frac{k-1}{v} \int_{0}^{T} \int_{\mathbb{R}} p_{k}\left|Q_{k}\right| \mathrm{d} x \mathrm{~d} t \\
\leq & C,
\end{aligned}
$$

where we have used Lemma 4.3.

We conclude that the sequence $\left(\phi_{k}\left(p_{k}\right)\right)_{k}$ converges strongly in $L^{2}((0, T) \times \mathbb{R})$. On the other hand, from the uniform bounds on $p_{k}$ we infer that $p_{k} \rightarrow p_{\infty}$, weakly in $L^{2}((0, T) \times$ $\mathbb{R})$, up to the subsequence. We can therefore apply Lemma 8.1 to conclude that

$$
\phi_{k}\left(p_{k}\right) \rightarrow p_{\infty}
$$

strongly in $L_{\text {loc }}^{2}((0, T) \times \mathbb{R})$. We claim that this in fact implies strong convergence of the sequence of pressures $\left(p_{k}\right)_{k}$ itself. Indeed, using the triangle inequality yields

$$
\left\|p_{k}-p_{\infty}\right\|_{L^{2}\left(0, T ; L^{2}(\mathbb{R})\right)} \leq\left\|p_{k}-\phi_{k}\left(p_{k}\right)\right\|_{L^{2}\left(0, T ; L^{2}(\mathbb{R})\right)}+\left\|\phi_{k}\left(p_{k}\right)-p_{\infty}\right\|_{L^{2}\left(0, T ; L^{2}(\mathbb{R})\right)},
$$

and

$$
\begin{aligned}
\int_{0}^{T} \int_{\mathbb{R}}\left|p_{k}-p_{k}^{\frac{k}{k-1}}\right|^{2} \mathrm{~d} x \mathrm{~d} t & =\int_{0}^{T} \int_{\mathbb{R}}\left|p_{k}\right|\left|\sqrt{p_{k}}-p_{k}^{\frac{1}{k-1}+\frac{1}{2}}\right|^{2} \mathrm{~d} x \mathrm{~d} t \\
& \leq \sup _{0 \leq z \leq p_{M}}\left|\sqrt{z}-\sqrt{z} z^{\frac{1}{k-1}}\right|^{2}\left\|p_{k}\right\|_{L^{1}\left(0, T ; L^{1}(\mathbb{R})\right)},
\end{aligned}
$$

with the right-hand side of the last line converging to zero. We conclude that

$$
p_{k} \rightarrow p_{\infty}
$$

strongly in $L_{\text {loc }}^{2}((0, T) \times \mathbb{R})$. In combination with the $L^{\infty}$-bounds, we deduce that this convergence holds strongly in $L_{\mathrm{loc}}^{p}\left(0, T ; L^{q}(\mathbb{R})\right)$, for any $2 \leq p, q<\infty$, using the dominated convergence theorem. Moreover, the convergence is also true almost everywhere. 


\section{Incompressible Limit and Complementarity Relation}

We have garnered all information necessary to pass to the incompressible limit in the pressure equation (4) and prove Theorem 2.2.

Proof of Theorem 2.2 Having established strong convergence of the sequence $\left(p_{k}\right)_{k}$, and weak convergence of $\left(n_{k}\right)_{k}$ due to the a priori estimates, we can pass to the limit in the relation

$$
n_{k} p_{k}=\left(\frac{k-1}{k}\right)^{\frac{1}{k-1}} p_{k}^{\frac{k}{k-1}},
$$

to deduce the relation $\left(1-n_{\infty}\right) p_{\infty}=0$, almost everywhere. For a test function $\varphi \in$ $C_{c}^{1}((0, T) \times \mathbb{R})$, let us recall the weak formulation of the equation for the pressure

$$
\begin{aligned}
\int_{0}^{T} \int_{\mathbb{R}} \frac{\partial \varphi}{\partial t} p_{k} & -\frac{\partial \varphi}{\partial x} p_{k} \frac{\partial W_{k}}{\partial x}-\varphi p_{k} \frac{\partial^{2} W_{k}}{\partial x^{2}} \mathrm{~d} x \mathrm{~d} t \\
& =-\int_{0}^{T} \int_{\mathbb{R}} \frac{k-1}{v} \varphi p_{k}\left[W_{k}-p_{k}+v r_{k} G^{(1)}\left(p_{k}\right)+v\left(1-r_{k}\right) G^{(2)}\left(p_{k}\right)\right] \mathrm{d} x \mathrm{~d} t .
\end{aligned}
$$

Due to the uniform bounds on the right-hand side, $c f$. Lemma 4.3, we may divide by $k-1$ to obtain

$$
0=\lim _{k \rightarrow \infty} \int_{0}^{T} \int_{\mathbb{R}} \varphi p_{k}\left[W_{k}-p_{k}+v r_{k} G^{(1)}\left(p_{k}\right)+v\left(1-r_{k}\right) G^{(2)}\left(p_{k}\right)\right] \mathrm{d} x \mathrm{~d} t .
$$

Note that, writing $n_{k}^{(1)}=n_{k} r_{k}$ and expressing $n_{k}$ in terms of $p_{k}$, in a fashion similar to Eq. (15), we may readily pass to the limit in all of these terms due to the strong convergence of the pressure and the a priori bounds of Lemma 4.1. We thus obtain

$$
0=p_{\infty}\left(W_{\infty}-p_{\infty}+v n_{\infty}^{(1)} G^{(1)}\left(p_{\infty}\right)+v n_{\infty}^{(2)} G^{(2)}\left(p_{\infty}\right)\right),
$$

in the weak sense, where $n_{\infty}^{(i)}$ satisfies

$$
\frac{\partial n_{\infty}^{(i)}}{\partial t}-\frac{\partial}{\partial x}\left(n_{\infty}^{(i)} \frac{\partial W_{\infty}}{\partial x}\right)=n_{\infty}^{(i)} G^{(i)}\left(p_{\infty}\right)
$$

for $i=1,2$. Indeed these equations follow by passing to the limit in the weak formulation of $(2 a),(2 b)$

$$
\int_{0}^{T} \int_{\mathbb{R}} \frac{\partial \varphi}{\partial t} n_{k}^{(i)}-\frac{\partial \varphi}{\partial x} n_{k}^{(i)} \frac{\partial W_{k}}{\partial x} \mathrm{~d} x \mathrm{~d} t=-\int_{0}^{T} \int_{\mathbb{R}} \varphi n_{k}^{(i)} G^{(i)}\left(p_{k}\right) \mathrm{d} x \mathrm{~d} t,
$$

where $\varphi \in C_{c}^{1}((0, T) \times \mathbb{R})$.

Remark 6.1 In fact, using the strategy of the previous section, i.e., the BV-bounds in space, in conjunction with a control on the time derivative obtained from bounding the right-hand side of the equation for the individual species, one can deduce also strong convergence of the sequence $\left(n_{k}\right)_{k}$. As a consequence, the limit functions $n_{\infty}, n_{\infty}^{(i)}$ are of bounded variation in time and space. 


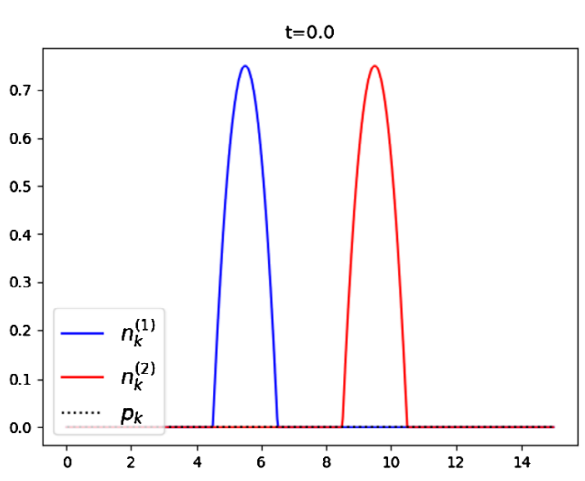

(a) Initial data, $\nu=1$.

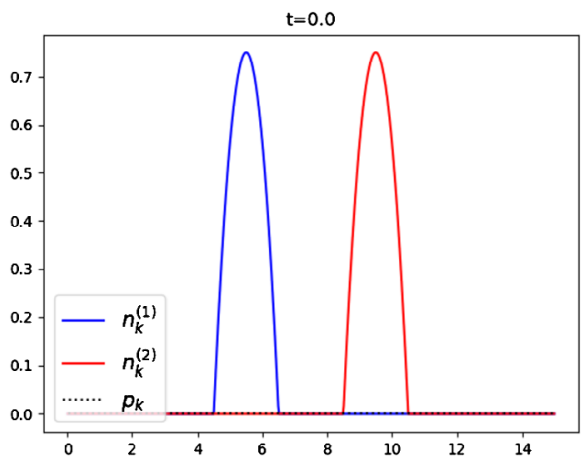

(c) Initial data, $\nu=0.01$.

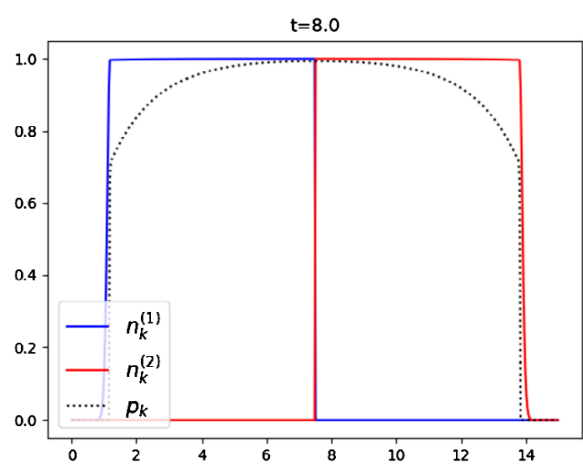

(b) Final time, $T=8$.

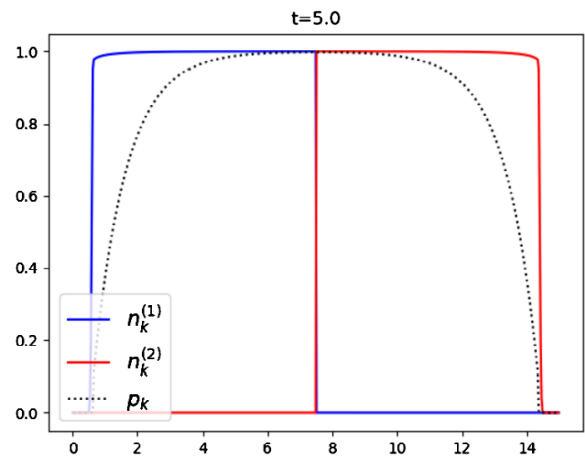

(d) Final time, $T=5$.

Fig. 1 We run the simulation for the same initial data for two different values of $v$, i.e., v=1 in the upper row and $v=0.01$ in the bottom row. In both cases, we chose $k=100$ since we are interested in the limiting behaviour. The individual species are represented by solid lines in red and blue, the pressure is superimposed as a black dotted line. In the upper row the pressure drops to zero immediately, whereas in the bottom row we can see an almost smooth transition

\section{Numerical Investigations}

In this section, we revisit the results from the preceding sections and showcase certain properties of the system. The numerical simulations are performed using the positivitypreserving upwind finite volume scheme proposed for a system of two interacting species in $[9,10]$ where the reaction terms are computed on each finite volume cell as simple ODEs. The implementation hinges on the fact that the elliptic Brinkman law (2b) can be solved using the integral representation (7).

Figure 1 displays the role of the viscosity parameter, $v$. The same initial data

$$
n_{k, 0}^{(1)}(x)=m(x-4.5)(6.5-x), \quad \text { and } \quad n_{k, 0}^{(2)}(x)=m(x-8.5)(10.5-x),
$$

are used in both cases and $m>0$ is chosen to normalise the initial mass to 1 . In both cases we used $k=100$, as we are interested in the incompressible regime. In addition, we chose $G^{(i)}(p)=1-p$, for $i=1,2$, corresponding to a homeostatic pressure of $p_{M}=1, c f$. Eq. (6). In both cases we observe the propagation of segregation in agreement with Lemma 4.2. Moreover, we observe a drastic drop in the pressure in Fig. 1(b). This was already observed 


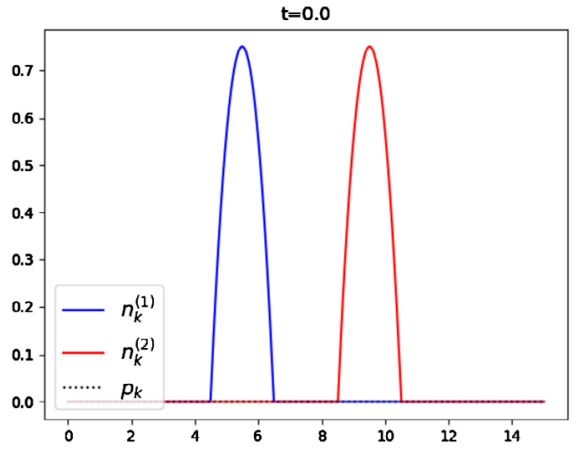

(a) Initial data, $t=0$.

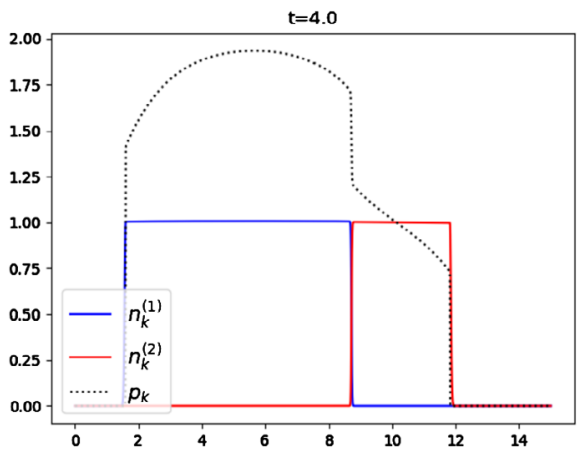

(c) Intermediate time, $t=4$.

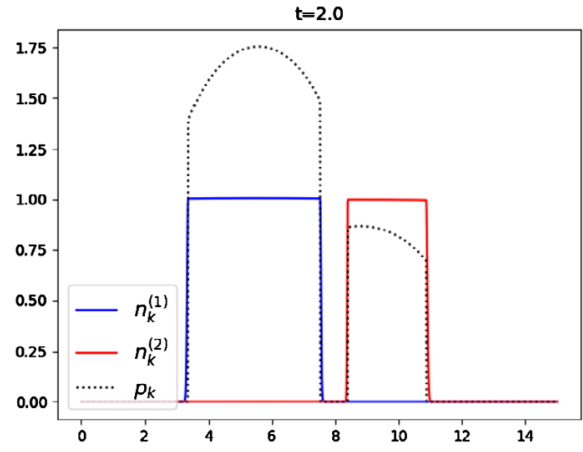

(b) Intermediate time, $t=2$.

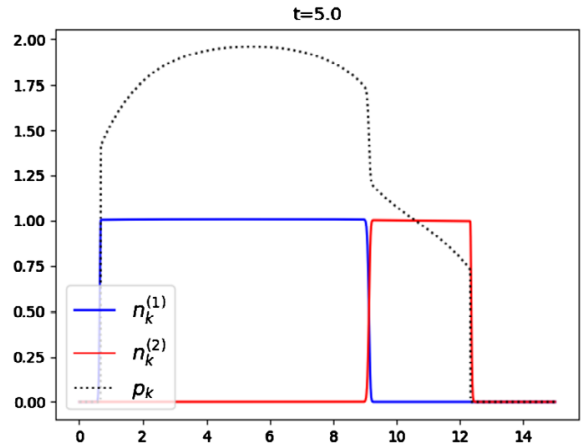

(d) Final time, $T=5$.

Fig. 2 We run the simulation for the same initial data for two different growth functions, $G^{(i)}(p)$. In both cases, we chose $k=100$ and the individual species are represented by solid lines in red and blue, the pressure is superimposed as a black dotted line, as before. The pressure drops not only at the boundary of its support. We also observe jumps in internal layers

in the one species case, $c f$. [19], where the fact was exploited that the limiting pressure has an integral representation formula. In stark contrast, Fig. 1(d) shows an almost smooth transition of the pressure indicating a much higher regularity. This is in perfect alignment with the findings of [6], as the case $v=0$ yields, at least formally, the system studied in the latter. As a matter of fact, the pressure gradient was shown to be square-integrable in the Darcy case, i.e., $v=0$. We conclude, by remarking that the front propagation is much faster in the regime of small $v$, another fact that was already observed in the single-species case.

In Fig. 2 we present the effect of different growth terms of the tumour cells and healthy tissue. To be more precise, we choose the same initial condition as above but use

$$
G^{(1)}(p)=2-p, \quad \text { and } \quad G^{(2)}(p)=1-p,
$$

as growth terms for the two species. We see that the first species, $n_{k}^{(1)}$, proliferates much faster compared to the second one. More interestingly, we see that the pressure not only has a jump at the boundaries of the support of the total population, but also at the internal layer. 


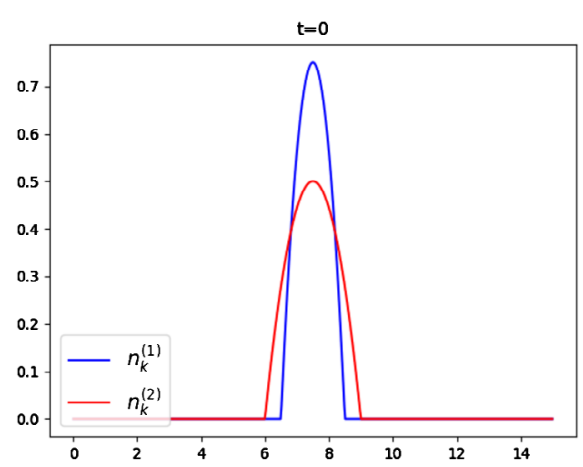

(a) Initial data, $t=0$.

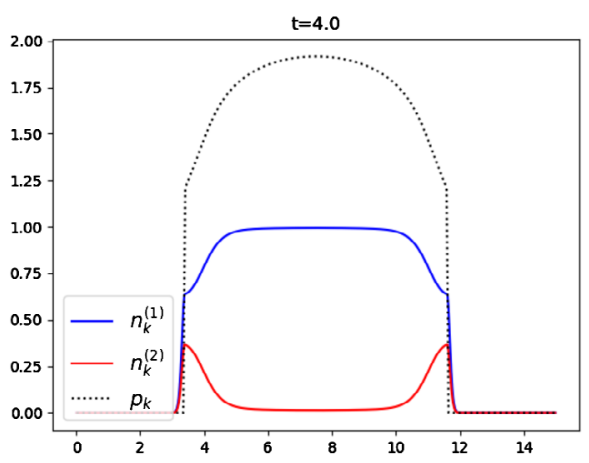

(c) Initial data, $t=4$

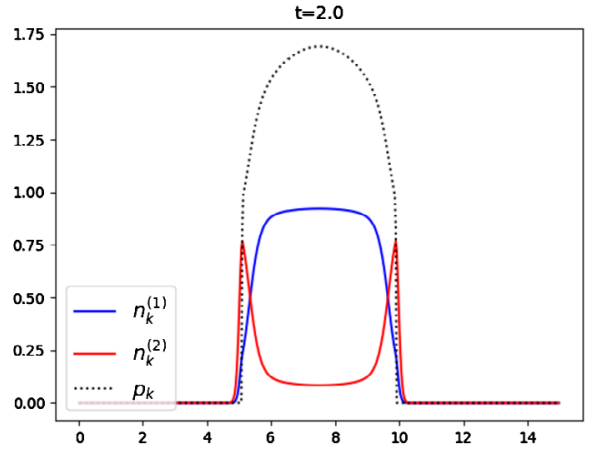

(b) Final time, $t=2$.

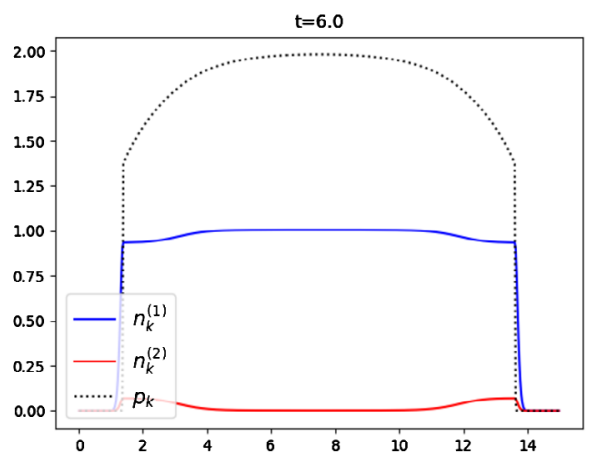

(d) Final time, $T=6$.

Fig. 3 The simulation shows the invasion of abnormal cells surrounded by healthy tissue. As time evolves, the tumour spreads and the density of normal cells is diminished and nearly vanishes, $c f$. Figure 3(d). As before, $v=1$ and $k=100$

Figure 3 shows the evolution of system (2a), (2b) for initial data representing a regime where healthy tissue has already been intruded by cancerous cells, i.e.,

$$
n_{k, 0}^{(1)}(x)=m(x-6.5)(8.5-x), \quad \text { and } \quad n_{k, 0}^{(2)}(x)=m(x-6)(9-x),
$$

where, again, $m>0$ normalises the mass. In addition, we choose the same unequal growth functions, $G^{(i)}$, as before, thus promoting the tumour growth compared to the normal tissue.

\section{Conclusions}

The goal of the paper was twofold. We extended an established tumour growth model to an interaction system of two cell populations, i.e., normal and abnormal cells. The interaction is given through the Brinkman flow, an elliptic equation that yields the velocity potentials for each cell population. In the first part of this paper we proved the existence of solutions to the interaction system, $c f$. Theorem 2.1. Building upon this result, we passed to the "incompressible" limit in the pressure equation, Eq. (4), and obtained the limiting equation, also referred to as complementarity relation, $c f$. Theorem 2.2. This way we were able to derive a geometric model from the cell-density model we presented. 
Note that both the existence result and the incompressible limit rely on strategies different from the ones adapted for related models (either in the parabolic two-species case when Brinkman's law is replaced by Darcy's law $(v=0)$ or the one-species model with Brinkman flow). The results are complemented with a numerical investigation showcasing the segregation result, the discontinuities in the pressure and the two individual population densities which is why we do not expect better regularity than bounded variation.

In summary, this paper extends known results in the literature to two species. As far as the existence of solutions is concerned, no additional difficulties are expected in the multidimensional case. However, when it comes to the stiff limit not only our method fails but also the kinetic reformulation that was employed in the one-species case, $c f$. [19], would need a serious make-over that is, at this stage, far from clear - even in one dimension. New singularities appear at internal layers when the two species meet and it seems that different tools are required, such as the extension of the kinetic reformulation to systems, which, to our knowledge, does not exist. The exploration of such a technique is left for future works.

In addition, the rigorous inviscid limit, $v \rightarrow 0$, remains an open question that is left for future work.

Acknowledgements The authors are grateful to Benoît Perthame and Nicolas Vauchelet for suggesting this problem and delightful discussions. This work was completed while T.D. was a visitor at Laboratoire JacquesLouis Lions, whose kind hospitality he appreciates. T.D. recognises the support of the Polish National Agency for Academic Exchange (NAWA) and National Science Center (Poland), grant no 2018/30/M/ST1/00423. M.S. fondly acknowledges the support of the Fondation Sciences Mathématiques de Paris (FSMP) for the postdoctoral fellowship.

Publisher's Note Springer Nature remains neutral with regard to jurisdictional claims in published maps and institutional affiliations.

Open Access This article is licensed under a Creative Commons Attribution 4.0 International License, which permits use, sharing, adaptation, distribution and reproduction in any medium or format, as long as you give appropriate credit to the original author(s) and the source, provide a link to the Creative Commons licence, and indicate if changes were made. The images or other third party material in this article are included in the article's Creative Commons licence, unless indicated otherwise in a credit line to the material. If material is not included in the article's Creative Commons licence and your intended use is not permitted by statutory regulation or exceeds the permitted use, you will need to obtain permission directly from the copyright holder. To view a copy of this licence, visit http://creativecommons.org/licenses/by/4.0/.

\section{Appendix}

For the readers' convenience we shall recall here the compactness method invoked in [15] in the context of the fast reaction limit in a cross-diffusion system with growth and death processes. Roughly speaking, it allows to identify the limit of the composition of a uniformly compact nonlinear function and a weakly convergent sequence.

Lemma 8.1 ("Lemma A") Let $\Omega \subset \mathbb{R}$ be a compact domain and set $Q_{T}:=(0, T) \times \Omega$. Furthermore, let $\left\{u_{n}\right\} \subset L^{\infty}\left(Q_{T}\right)$ and $\left\{f_{n}\right\} \subset C(\mathbb{R})$ be sequences with the properties

(i) $u_{n} \rightarrow u$, weakly in $L^{2}\left(Q_{T}\right)$,

(ii) $f_{n}$ is nondecreasing,

(iii) $f_{n} \rightarrow f$, uniformly on compact subsets of $\mathbb{R}$, and

(iv) $f_{n}\left(u_{n}\right) \rightarrow \chi$, strongly in $L^{2}\left(Q_{T}\right)$. 
Then

$$
\chi=f(u) .
$$

For the sake of exposition, we recall here that the assumptions of the above lemma are indeed met in our case.

Remark 8.2 (The assumptions are met) The first assumption is the easiest to check as it follows directly from the uniform $L^{\infty}$-bounds on the pressure. Similarly, it is readily verified that each element of the sequence of functions, in our context given by $\phi_{k}(x)=x^{k / k-1}$, is indeed nondecreasing. Moreover, the uniform convergence towards the identity is straightforward. Thus the only requirement that needs a more minute argument is (iv) which we present in the first part of the proof of Lemma 5.3.

\section{References}

1. Allaire, G.: Homogenization of the Navier-Stokes equations and derivation of Brinkman's law. In: Mathématiques appliquées aux sciences de l'ingénieur, Santiago, 1989, pp. 7-20. Cépaduès, Toulouse (1991)

2. Bertsch, M., Gurtin, M.E., Hilhorst, D.: On a degenerate diffusion equation of the form $c(z)_{t}=\varphi\left(z_{x}\right)_{x}$ with application to population dynamics. J. Differ. Equ. 67(1), 56-89 (1987)

3. Bertsch, M., Gurtin, M.E., Hilhorst, D.: On interacting populations that disperse to avoid crowding: the case of equal dispersal velocities. Nonlinear Anal., Theory Methods Appl. 11(4), 493-499 (1987)

4. Bertsch, M., Gurtin, M.E., Hilhorst, D., Peletier, L.A.: On interacting populations that disperse to avoid crowding: preservation of segregation. J. Math. Biol. 23(1), 1-13 (1985)

5. Bertsch, M., Hilhorst, D.A., Izuhara, H., Mimura, M.: A nonlinear parabolic-hyperbolic system for contact inhibition of cell-growth. Differ. Equ. Appl. 4(1), 137-157 (2012)

6. Bubba, F., Perthame, B., Pouchol, C., Schmidtchen, M.: Hele-Shaw limit for a system of two reaction(cross-)diffusion equations for living tissues. Arch. Ration. Mech. Anal. (2019)

7. Byrne, H., Drasdo, D.: Individual-based and continuum models of growing cell populations: a comparison. J. Math. Biol. 58(4-5), 657-687 (2009)

8. Carrillo, J.A., Fagioli, S., Santambrogio, F., Schmidtchen, M.: Splitting schemes and segregation in reaction cross-diffusion systems. SIAM J. Math. Anal. 50(5), 5695-5718 (2018)

9. Carrillo, J.A., Filbet, F., Schmidtchen, M.: Convergence of a Finite Volume Scheme for a System of Interacting Species with Cross-Diffusion. ArXiv e-prints (2018)

10. Carrillo, J.A., Huang, Y., Schmidtchen, M.: Zoology of a nonlocal cross-diffusion model for two species. SIAM J. Appl. Math. 78(2), 1078-1104 (2018)

11. Chertock, A., Degond, P., Hecht, S., Vincent, J.-P.: Incompressible limit of a continuum model of tissue growth with segregation for two cell populations. ArXiv e-prints (2018)

12. Degond, P., Hecht, S., Vauchelet, N.: Incompressible limit of a continuum model of tissue growth for two cell populations. Netw. Heterog. Media 15(1), 57-85 (2020)

13. Gwiazda, P., Perthame, B., Świerczewska-Gwiazda, A.: A two-species hyperbolic-parabolic model of tissue growth. Commun. Partial Differ. Equ. 44(12), 1605-1618 (2019)

14. Hecht, S., Vauchelet, N.: Incompressible limit of a mechanical model for tissue growth with nonoverlapping constraint. Commun. Math. Sci. 15(7), 1913 (2017)

15. Hilhorst, D., van der Hout, R., Peletier, L.A.: Nonlinear diffusion in the presence of fast reaction. Nonlinear Anal., Theory Methods Appl. 41(5-6), 803-823 (2000)

16. Mellet, A., Perthame, B., Quirós, F.: A Hele-Shaw problem for tumor growth. J. Funct. Anal. 273(10), 3061-3093 (2017)

17. Perthame, B., Quirós, F., Tang, M., Vauchelet, N.: Derivation of a Hele-Shaw type system from a cell model with active motion. Interfaces Free Bound. 16, 489-508 (2014)

18. Perthame, B., Quirós, F., Vázquez, J.L.: The Hele-Shaw asymptotics for mechanical models of tumor growth. Arch. Ration. Mech. Anal. 212(1), 93-127 (2014)

19. Perthame, B., Vauchelet, N.: Incompressible limit of a mechanical model of tumour growth with viscosity. Philos. Trans. R. Soc. A 373, 20140283, 16 (2050). 2015

20. Ranft, J., Basan, M., Elgeti, J., Joanny, J.-F., Prost, J., Jülicher, F.: Fluidization of tissues by cell division and apoptosis. Proc. Natl. Acad. Sci. 107(49), 20863-20868 (2010) 